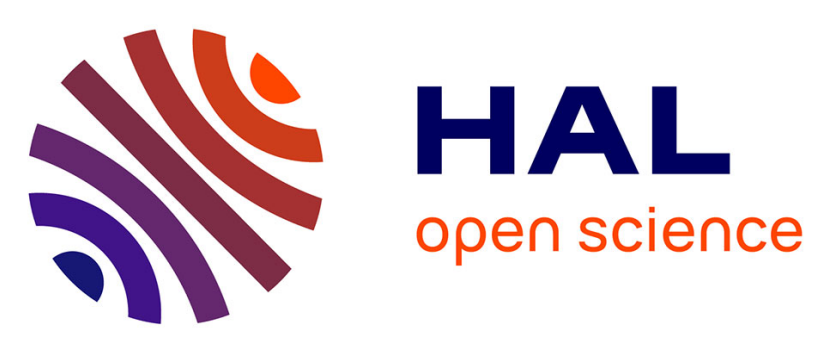

\title{
Parameter identification of health indicator aggregation for decision-making in predictive maintenance: Application to machine tool
}

\author{
Thomas Laloix, Benoît Iung, Alexandre Voisin, Eric Romagne
}

\section{- To cite this version:}

Thomas Laloix, Benoît Iung, Alexandre Voisin, Eric Romagne. Parameter identification of health indicator aggregation for decision-making in predictive maintenance: Application to machine tool. CIRP Annals - Manufacturing Technology, 2019, 68 (1), pp.483-486. 10.1016/j.cirp.2019.03.020 . hal-02125367

\section{HAL Id: hal-02125367 \\ https://hal.science/hal-02125367}

Submitted on 10 May 2019

HAL is a multi-disciplinary open access archive for the deposit and dissemination of scientific research documents, whether they are published or not. The documents may come from teaching and research institutions in France or abroad, or from public or private research centers.
L'archive ouverte pluridisciplinaire HAL, est destinée au dépôt et à la diffusion de documents scientifiques de niveau recherche, publiés ou non, émanant des établissements d'enseignement et de recherche français ou étrangers, des laboratoires publics ou privés. 


\title{
Parameter identification of health indicator aggregation for decision-making in predictive maintenance: Application to machine tool
}

\author{
Thomas Laloix (3) $)^{\mathrm{a}, \mathrm{b}}$, Benoit Iung (1) ${ }^{\mathrm{a}, *}$, Alexandre Voisin ${ }^{\mathrm{a}}$, Eric Romagne ${ }^{\mathrm{b}}$ \\ a Université de Lorraine, CRAN, UMR CNRS 7039, Campus Sciences, BP 70239, Vandœuvre-lès-Nancy, 54506, France \\ ${ }^{\mathrm{b}}$ Renault, Usine de Cléon, BP 105, Cléon, 76410, France
}

\section{A R T I C L E I N F O}

\section{Keywords:}

Maintenance

Decision-making

Multi-level modeling

\begin{abstract}
A B S T R A C T
In predictive maintenance, an essential step entails anticipative decision-making to avoid industrial system failure. Nevertheless, decision-making is often based on a component status indicator or information fusion dedicated to a specific application. It leads to a decision most of the time not enough relevant in regards with system consideration as a whole. An interesting approach is to propose a global view of health state of multi-level system to decision-makers by considering indicators at a given level and their interactions for aggregation to obtain indicators at the upper level. For this purpose, the most suited operator is the Choquet integral that is already used at the single level. Furthermore, parameter identification of aggregation operator should consider the multi-level issue, including the cumulative error between the levels. As such, conventional identification approaches do not address this multi-level issue. To control this problem, an improvement of a conventional identification algorithm based on genetic algorithm is proposed. The improvement is related to Choquet integral parameter identification considering multi-level cumulative error. The application of this improved operator is illustrated in the Renault context, on a machine tool producing cylinder blocks.
\end{abstract}

(C) 2019 Published by Elsevier Ltd on behalf of CIRP.

\section{Introduction}

In manufacturing, maintenance is an engineering service that aims to guarantee the performance of production equipment throughout the service-life to boost profitability [1]. This postulate is particularly true in the case of predictive maintenance, which promotes optimal maintenance scheduling and production quality based on an ability to anticipate service-related issues [2]. The relationship between manufacturing system maintenance and the required delivered quality should lead to a reduction of a posteriori quality control [3]. This predictive maintenance vision is expected in Renault factories (e.g. power train factory of Cléon in France), where predictive maintenance is applied to machine tools to achieve product quality with a diminution of a posteriori quality control (e.g. SPC method).

An essential step in the implementation of such a strategy entails providing system health state to decision-makers to pilot and maintain manufacturing production and equipment. System health necessarily constitutes performance and degradation indicators, rather than raw data, related to physical phenomenon

\footnotetext{
* Corresponding author.

E-mail address: benoit.iung@univ-lorraine.fr (B. Iung).
}

[4]. This requires the transformation of monitored parameters provided by the physical data from sensors, into information represented as indicators. The potential benefits do not only include the reduction of the quantity of data examined, but also the maximization of the useful information content obtained from relationships between indicators at a given hierarchical level [5].

However, with regard to the system abstraction levels, indicators should be associated with each abstraction level thanks to aggregation operators. The resulting evaluation of these indicators will then differ according to selected aggregation operators. In this way, the consideration of multi-level dimensions for performance indicators is often performed by physical law [6]. Other forms of aggregation operators are required for degradation indicators to model the impact that degradation at a given level has on the upper levels. Such aggregation has to consider indicator interactions [7]. Well relevant operator that offers the ability to integrate interactions between indicators is the Choquet integral. For practical use, it is necessary to identify its parameter. Indeed, parameter identification for aggregation in a multi-level system should consider the system as a whole and the cumulative error between the levels. This error is not considered by the conventional identification methods, which mainly focused on a single level and does not address the problem statement of multi-level system. In this way, this paper proposes an original 
approach to tackle the multi-level identification issue by improvement of the Choquet integral parameter identification for multi-level aggregation through the use of genetic algorithms. The Renault case focusing on multi-level indicators of a machine tool serves as use case.

To explain the basis for this improvement, Section 2 introduces the concept of health indicator evaluation for multi-level systems. Section 3 focuses on the development of the Choquet integral parameter identification by means of a genetic algorithm. Section 4 illustrates this development in the machine-tool context of a Renault factory. Finally, conclusion and future work are summarized in Section 5.

\section{Problem statement on health indicator elaboration for multi-level systems}

In the field of decision theory, information aggregation is distinguished from information fusion since it is considered to refer to concrete mathematical functions [8]. Such methods have demonstrated their utility in the case of indicator combination for health check evaluation [4]. Aggregation functions (or operators) aim to summarize the information contained in an n-tuple of input values by means of a single representative output value. These functions take arguments from the closed interval [0,1] and produce a real value in $[0,1]$, usually without loss of generality. This is usually denoted as $f:[0,1] n \rightarrow[0,1]$ for a function with an argument consisting of $n$ inputs [9].

Among aggregation operators, Choquet integral appears to be the most suitable for considering the interaction between indicators, as required by the multi-level situation [8].

\subsection{Choquet integral and associated capacities}

Indeed, the Choquet integral is based on a capacity [10]. Let $S:=$ $\{1, \ldots, \mathrm{n}\}$ be a finite set. A capacity $\mu: \wp(S) \rightarrow[0,1]$ defined on the set of the subsets of $S$, i.e. the power set of $S, \wp(S)$, must satisfy the following conditions:

$$
\left\{\begin{array}{c}
\mu(\emptyset)=0 \\
\mu(S)=1 \\
\mu(A) \leq \mu(B), \forall A \subseteq B \subseteq S
\end{array}\right.
$$

From this capacity $\mu$, the Choquet integral of the set of indicator $\{X\}:=\left\{x_{1}, \ldots, x_{n}\right\}$ is defined as:

$C_{\mu}\left(x_{1}, \cdots, x_{n}\right)=\sum_{i=1}^{n}\left(x_{(i)}-x_{(i-1)}\right) \mu\left(A_{(i)}\right)$

where ${ }_{(.)}$used in the sub-script, is a permutation operator such that: $x_{(1) \leq} \ldots \leq x_{n}$ and $A_{(i)}:\left\{x_{(i)}, \ldots, x_{(n)}\right\}$ with $x_{(0):}=0$; and $\mu\left(A_{(i)}\right)$ capacity value of $A_{(i)}$.

The capacity coefficient can be interpreted as the weights of a weighted mean over the power set of $X$. Indeed, $\mu(A)$ represents the degree of importance of $A \subseteq X$ with regard to the value of $X$. Then, the boundary condition implies that the empty set, with the absence of any indicators, has no importance, i.e. $\mu(\varnothing)=0$ and the entire set, i.e. the presence of all indicators, has maximum importance where $\mu$ $(X)=1$ [11]. Moreover, the monotonicity condition implies that the addition of a new indicator to a combination of subsets cannot decrease its importance. The distinctiveness of capacities relies on their ability to represent the interaction between indicators, in addition to their relative importance. Consider $A$ and $B$ as two subsets of indicators where $A \cap B=\emptyset$. The interaction of the two indicators subsets could be: no interaction leading to $\mu\{A \cup B\}=\mu\{A\}+\mu\{B\}$; negative interaction leading to $\mu\{A \cup B\}<\mu\{A\}+\mu\{B\}$; or positive interaction leading to $\mu\{A \cup B\}>\mu\{A\}+\mu\{B\}$.

Thus, the capacity value of an indicator subset reflects the interaction between its elements. For instance, in the machine tool domain, a lack of lubricant and an unbalance spindle interact synergistically to produce spindle degradation, whereas bearing wear and nut wear of a linear axis interacts redundantly because this combination does not lead to a more important impact on the axis. In practical application the major challenge is to identify the capacity.

\subsection{Capacity identification for a single-level system ... towards multi-level system}

Indeed, capacity identification constitutes a drawback in the practical use of the Choquet integral, particularly in the case of a large system with an important number of inputs. Indeed, the number of parameters to be identified for each capacity, with $n$ indicators, is $2^{n}-2[10,12]$.

In this way, most of the methods for capacity identification can be stated as optimization problems. For example, in Ref. [12], four main approaches were identified such as the least squares-based approach, or the maximum split approach. Nevertheless, the usage of such methods is limited, because they require initial inputs or information on the desired overall performance score which cannot be easily or accurately provided by the decision makers [11].

These limits are constraints in attempting to support multi-level capacity identification issue, leading to the consideration of alternative approaches, such as those based on genetic algorithm (GA). This is because these approaches are relevant to supporting optimization due to their simplicity of implemented and the flexibility to express the optimization function. This technique was successfully applied by Ref. [10] as an optimization method to identify the capacity that best fits a set of data, with the squared error as a criterion of fitness. This approach entails the use of convex combination of capacities as a crossover operator coupled with the setting of the initial population in the set of extreme points of the capacity space, i.e. the binary capacity set. Nevertheless, identification of the capacities is performed only for a single level and does not address multi-level capacity identification where the output error has to be chained. This is because a multi-level system can be considered as "chained" single-level systems (e.g. propagation of the modeling error needs to be considered).

For this multi-level issue of the capacity identification, Ref. [4] developed the identification approach from the principle of bottom-up capacity inference. Indeed, in Ref. [4], data are only available at the component level, which allowed the capacities of the upper element to be inferred from the capacities of the related sub-elements at the lower level. However, the hypothesis of this paper is different since monitored indicators (i.e. machine tool industrial case) are available at all the system levels. It facilitates, in an original way, the identification of the capacity of each element of the system. Nevertheless, two approaches can be considered. The first one is called local identification and uses existing identification algorithms to independently determine the capacity for every element. The second one called global identification aims to simultaneously identify the capacities of all elements in a holistic view. This is achieved by minimization of the error due to successive use of aggregation along the system hierarchy.

Our scientific proposal addresses the second approach in an original manner by facilitating the tailoring of the GA-based method to support optimization.

\section{Proposition of Choquet integral capacity identification in health indicator evaluation for multi-level-systems}

Our contribution is therefore based on the formulation of the global error computation for multi-level systems and multi-level capacity identification using genetic algorithms. The proposal relevance is assessed via a comparison with local optimization.

\subsection{Local vs "chained" computation}

As an example of a comparison between both local and global identification approaches, a sufficiently generic system structure (Fig. 1) is applied. Therefore, it is considered that a system $S$ is composed of 3 sub-systems, $\left\{\mathrm{S}_{1}, \mathrm{~S}_{2}, \mathrm{~S}_{3}\right\}$, each of which is composed 


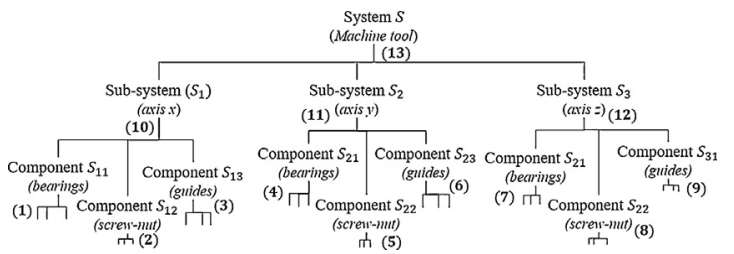

Fig. 1. Ad hoc manufacturing system structure.

of 3 components $\left\{S_{11}, S_{12}, S_{13}\right\},\left\{S_{21}, S_{22}, S_{23}\right\}$, and $\left\{S_{31}, S_{32}, S_{33}\right\}$. It is noted that $\Psi=\left\{S, S_{1}, S_{11}, S_{12}, S_{13}, S_{2} \ldots\right\}$ the set of all elements.

For every component, 3 indicators are introduced, e.g. for $S_{11}$ the set of indicators is $\left\{\mathrm{I}_{111}, \mathrm{I}_{112}, \mathrm{I}_{113}\right\}$. Hence, when considering indicators at all levels, the computation from low-level indicators up to system level one gives rise to a set of equations. The equation system clearly shows the nested relation that exists between the levels of the system:

$$
\left\{\begin{array}{c}
I_{S}=\operatorname{Aggr} v\left(I_{S_{1}}, I_{S_{2}}, I_{S_{3}}\right) \\
I_{S_{k}}=\operatorname{Aggr}_{S_{k}}^{v}\left(I_{S_{k 1}}, I_{S_{k 2}}, I_{S_{k 3}}\right), \forall k \in\{1,2,3\} \\
I_{S_{k l}}=\operatorname{Aggr}_{S_{k}}^{v}\left(I_{S_{k 11}}, I_{S_{k \mid 1}}, I_{S_{k 13}}\right), \forall k \in\{1,2,3\}, l \in\{1,2,3\} .
\end{array}\right.
$$

In the way of focusing on the error computation of the optimization, it is then considered a learning set of data, represented by $\mathrm{I}^{\mathrm{d}}$. The local error is based on the error for every element considered independently:

$$
\left\{\begin{array}{c}
E r_{S}^{L}=I_{S}^{d}-A \hat{g g} r_{S}^{v}\left(I_{S_{1}}^{d}, I_{S_{2}}^{d}, I_{S_{3}}^{d}\right) \\
E r_{S_{k}}^{L}=I_{S_{k}}^{d}-A g g r_{S_{k}}^{v}\left(I_{S_{k 1}}^{d}, I_{S_{k 2}}^{d}, I_{S_{k 3}}^{d}\right), \forall k \in\{1,2,3\} \\
E r_{S_{k l}}^{L}=I_{S_{k l}}^{d}-A g \hat{g} r_{S_{k l}^{v}}^{v}\left(I_{S_{k l 1}}^{d}, I_{S_{k \mid 2}}^{d}, I_{S_{k 13}}^{d}\right), \forall k \in\{1,2,3\}, l \in\{1,2,3\}
\end{array}\right.
$$

These sets of equations can easily be used in existing identification algorithms as presented in Section 2.2. For each element, capacity can be identified independently.

However, the global error considers aggregation propagation through multi-level layers of the system in a holistic way and is defined as:

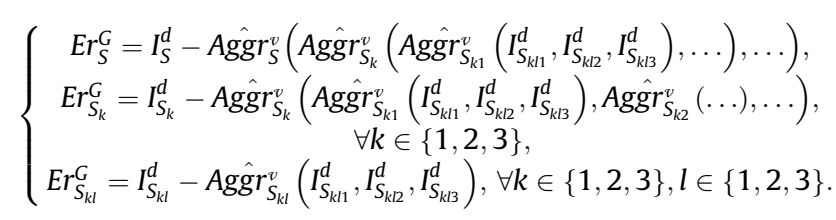

As shown in the error equations, the concept of "chained" computation materializes with the multi-level dependency in the indicator aggregation as shown in Eq. (3). This leads to a different optimization function that considers the propagation of the error toward the hierarchy of the system elements.

The global error computation should then be used in the fitness function of the genetic algorithm. The chosen method is adapted from [10] and should allow a comparison with the original method used for local optimization.

\subsection{Genetic algorithm construction for global capacity identification}

To fit with the global optimization model of capacity identification, the genetic algorithm needs to be adapted.

Indeed, in compliance with Ref. [10], the "local" optimization problem can be stated as the identification of the capacities that minimize the squared error. The squared error (i.e. Quadratic Error $Q E)$ to be minimized in the local approach, i.e. fitness function of $\mathrm{GA}$, is:

$Q E(X)=\sum_{i=1}^{n}\left(E_{X}^{L}\left(x_{X}^{i}\right)\right)^{2}$

where $x_{X}^{i} \in I^{d}$ and $\mathrm{n}$ is the number of learning dataset points.

For the global identification problem, the error computation must be adapted. Given that the optimization is performed for the system as a whole, i.e. considering all the capacities in the same identification process, the fitness function is the weighted sum of global errors, expressed in Eq. (5), and is called Nested Quadratic Error (NQE) defined as follows:

$N Q E=\sum_{X \in \Psi} \omega_{X} \sum_{i=1}^{n}\left(E_{X}^{G}\left(x_{X}^{i}\right)\right)^{2}$,

where, $\omega_{X}, X \in \Psi$, are the weights that provided balance between upper levels, where the number of elements is smaller, and lower levels, where number of elements is greater.

The question of the validation of the global approach including $\mathrm{NQE}$ then arises. Hence, it is compared to local approach to determine the best approach for capacity identification in the process of system health check evaluation and more precisely, in health check evaluation. Thus, context of a machine tool is used as a case study for local/global approaches comparison.

\section{Case study based on machine tool consideration}

At this stage, it is necessary to illustrate the application of the proposed approach in a real case study of a Renault context to compare both local and global capacity identification approaches on 5-axis machine tool called GROB BZ560. Therefore, only the linear axes are considered for the sake of demonstration. In relation to Fig. 1, this means that the machine tool system (13 in Fig. 1 ) is composed of three linear axis sub-systems $(10,11,12$ in Fig. 1), each consisting of three components (bearing, screw-nut, and guides) (1, 2, 3; 4, 5, 6; 7, 8, 9 in Fig. 1). Each component possesses 3 degradation indicators, whose values originate from the monitoring system (e.g. vibration, position error).

In order to examine the robustness of the algorithm, the learning set $\mathrm{I}^{\mathrm{d}}$ is "contaminated" with noise. The magnitude of the noise, the number of learning points and the population size of GA are adjusted to facilitate sensitivity analyses. The values of the parameters selected for the experiment are summarized in Table 1.

Table 1

Set of variation for experiment parameters.

\begin{tabular}{llll}
\hline Parameter & Values & & \\
\hline Noise & 0.10 & 0.15 & 0.20 \\
Sample & 20 & 50 & 100 \\
Pop. size Global & 10000 & 50000 & 100000 \\
Pop. size Local & 3800 & 10000 & \\
\hline
\end{tabular}

To evaluate the relevance of both approaches, the NQE (Eq. (5)) is considered with weighting vector equal to 1 for all elements. It will be referred to as a nested quadratic error. For the global fitness function of GA, a weighting vector is chosen with a factor 1 for all components, a factor 2 for sub-systems, and a factor 3 for system. The results of the experiments presented in Table 2 for 20 learning points is representative of the experiment performed with 50 points and 100 points. This clearly shows that the NQE between the global capacity identification and local one depends of the level of noise. Indeed, whatever the parameters of the experiment (see Table 1), only noise influences the comparison between the two

Table 2

Approximation error for 20 points.

\begin{tabular}{llll}
\hline Experiments & 1 & 2 & 3 \\
\hline Noise & 0.20 & 0.15 & 0.10 \\
Points & 20 & 20 & 20 \\
Pop. size Local & 5000 & 3800 & 5000 \\
Pop. size Global & 50000 & 50000 & 50000 \\
NQE for local GA & 0.611 & 0.317 & 0.158 \\
NQE for global GA & 0.562 & 0.375 & 0.218 \\
Time local & 588 & 654 & 648 \\
Time global & 7290 & 18800 & 10900 \\
\hline
\end{tabular}


methods of identification. For instance, the NQE global is lower for a higher noise level.

The simulation duration of the global optimization is between 2 and $7 \mathrm{~h}$ whereas the simulation duration of the local optimization is approximately ten of minutes. This can be explained based on the number of parameters that need to be identified at each optimization iteration. Indeed, it represents the local identification approach $2^{n}$, with $n=3$, whereas it is $13^{*} 2^{n}$ for global identification. This requires a much larger population size. It entails longer computation times at each generation and more generation to converge.

Nevertheless, when considering the experiment at the different abstraction levels, as illustrated Fig. 2 for experiment 1, in Table 2, with 20 points and $20 \%$ standard deviation of the noise, it appears that global identification is better for sub-system and system level whereas the local identification is better at the component level. Regarding the scale of the error, the difference in the errors between the local and global at the component level is too high, i.e. about 1.5 , to be compensated for at the sub-system and system level, i.e. respectively approximately 0.4 and 0.25 . This is explained by the higher number of components (i.e. 9 in the considered system) compared to the upper levels (i.e. 4, 3 sub-systems and 1 system). Remember that for this comparison, the weighted

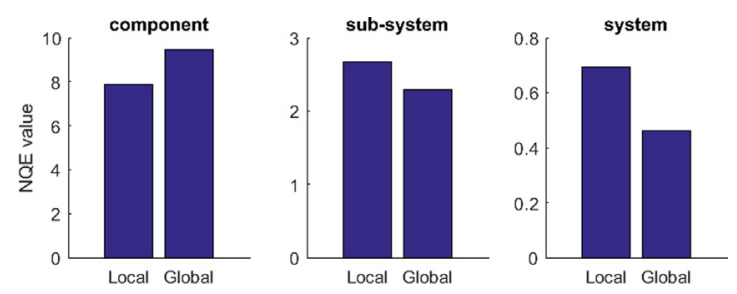

Fig. 2. NQE contribution by abstraction level for experiment 1, Table 2.

vector $\omega_{X}$ in Eq. (5) is set to 1 for all elements.

This experiment is particularly interesting in the sense that it represents a configuration, in a real application, where an expert (or a group of experts) defines a set of values of indicators depending on their relationship, leading to the availability of few data with large noise. When considering the error at the component level, as illustrated in Fig. 3, it can be noted that the global error is systematically higher than the local one (from 1 to 9). At the sub-system (linear axis) and system (machine tool)

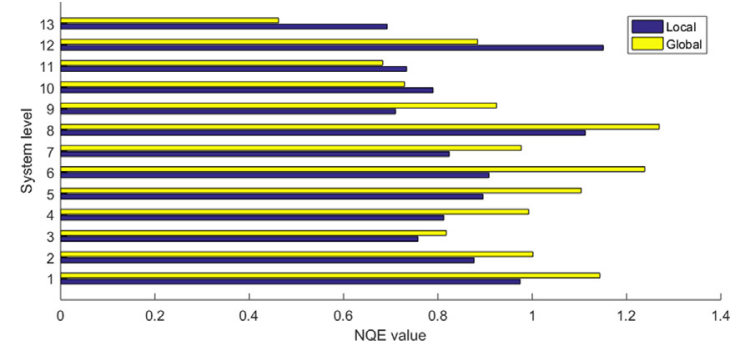

Fig. 3. NQE contribution of every element of the system for experiment 1 , Table 2 .

levels, the global error is systematically lower than the local one.

In order to illustrate the contribution of the NQE, Fig. 4 presents a comparison between the QE, Eq. (4), of the sub-systems (linear axis) and that of the system (machine tool), and the NQE contribution at the same levels.

In relation to the machine tool case, the results imply that the confidence level of the capacity identification for decision-making is higher in the sub-system level and system level than the component level, with global optimization. This conclusion is relevant because in the machine tool application case, and global manufacturing systems, indicators are in relation from one to another between system abstraction levels. Thus, the global approach has some interests in capacity identification due to its
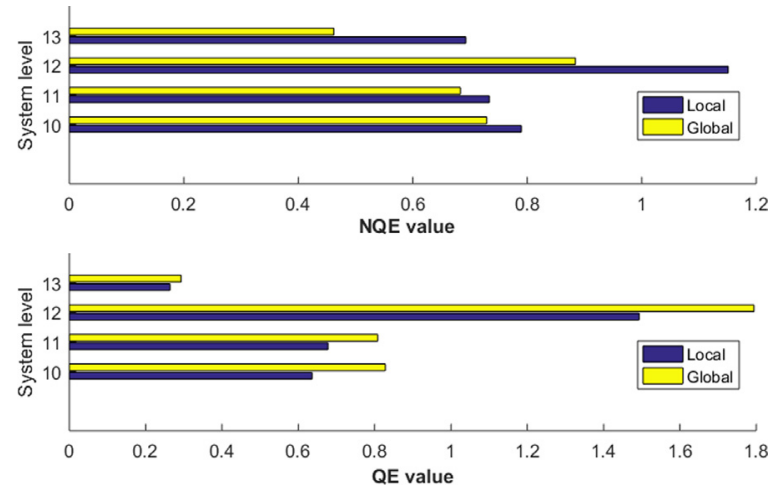

Fig. 4. $Q E$ and NQE values for sub-systems and system for local and global optimization (experiment 1, Table 2).

ability to simultaneously handle capacity identification of the entire system while integrating its limits in performance at the component level (towards a combination of the two approaches).

\section{Conclusions}

The paper presents an approach for Choquet integral capacity identification in the case of multi-level indicator aggregation for decision-making. The multi-level parameter identification relies on a global optimization model based on a genetic algorithm. An ad hoc system representative of a manufacturing multi-level system serves as an illustration to demonstrate the applicability of the approach. The results of the experiment indicate that a relevance perspective should be the combination of both a local and global optimization model for minimizing the modeling error for multilevel indicator parameter identification.

\section{Acknowledgments}

High Performance Computing resources were partially provided by the EXPLOR center hosted by the University de Lorraine.

\section{References}

[1] Roy R, Stark R, Tracht K, Takata S, Mori M (2016) Continuous Maintenance and the Future-Foundations and Technological Challenges. CIRP Annals 65:667688

[2] Gao R, Wang L, Teti R, Dornfeld D, Kumara S, Mori M, Helu M (2015) CloudEnabled Prognosis for Manufacturing. CIRP Annals - Manufacturing Technology 64:749-772.

[3] Colledani M, Tolio T, Fischer A, Iung B, Lanza G, Schmitt R, Váncza J (2014) Design and Management of Manufacturing Systems for Production Quality. CIRP Annals - Manufacturing Technology 63:773-796.

[4] Abichou B, Voisin A, Iung B (2015) Choquet Integral Capacity Calculus for Health Index Estimation of Multi-Level Industrial Systems. IMA Journal of Management Mathematics 26:205-224.

[5] Abichou B, Voisin A, Iung B (2012) Choquet Integral Parameters Inference for Health Indicators Fusion Within Multi-Levels Industrial Systems: Application to Components in Series. IFAC Proceedings 45:193-198.

[6] Panetto H, Pétin J (2005) Metamodelling of Production Systems Process Models Using UML Stereotypes. International Journal of Internet and Enterprise Management 3(2):155-169.

[7] Voisin A, Laloix T, Iung B, Romagne E (2018) Predictive Maintenance and Part Quality Control From Joint Product-Process-Machine Requirements: Application to a Machine Tool. Procedia Manufacturing 16:147-154.

[8] Torra V (2013) Aggregation Operators. On Fuzziness, Springer, Berlin Heidelberg691-695.

[9] Beliakov G, Pradera A, Calvo T (2007) Aggregation Functions: A Guide for Practitioners.

[10] Combarro EF, Miranda P (2006) Identification of Fuzzy Measures from Sample Data with Genetic Algorithms. Computers \& Operations Research 33:30463066.

[11] Krishnan AR, Kasim MM, Bakar EMNEA (2015) A Short Survey on the Usage of Choquet Integral and its Associated Fuzzy Measure in Multiple Attribute Analysis. Procedia Computer Science 59:427-434.

[12] Grabisch M, Kojadinovic I, Meyer P (2008) A Review of Methods for Capacity Identification in Choquet Integral Based Multi-Attribute Utility Theory. European Journal of Operational Research 186:766-785. 